\title{
Spectral line width decrease in the solar corona: resonant energy conversion from Alfvén to acoustic waves
}

\author{
T. V. Zaqarashvili ${ }^{1,2}$, R. Oliver ${ }^{1}$, and J. L. Ballester ${ }^{1}$ \\ 1 Departament de Física, Universitat de les Illes Balears, 07122 Palma de Mallorca, Spain \\ e-mail: [temury.zaqarashvili; ramon.oliver; dfsjlbo]@uib.es \\ 2 Georgian National Astrophysical Observatory (Abastumani Astrophysical Observatory), Kazbegi Ave. 2a, Tbilisi 0160, Georgia \\ e-mail: temury@genao.org
}

Received 12 June 2006 / Accepted 19 July 2006

\begin{abstract}
Context. Observations reveal an increase with height of the line width of several coronal spectral lines probably caused by outwardly propagating Alfvén waves. However, the spectral line width sometimes shows a sudden decrease at a height $\sim 0.1-0.2 R_{\odot}$, where the ratio of sound to Alfvén speeds may approach unity.

Aims. To explain the observed line width reduction in terms of the energy conversion from Alfvén to another type of wave motion. Methods. Weakly non-linear wave-wave interaction in ideal MHD.

Results. Qualitative analysis shows that the resonant energy conversion from Alfvén to acoustic waves near the region of the corona where the plasma $\beta$ approaches unity may explain the observed spectral line width reduction.
\end{abstract}

Key words. Sun: corona - Sun: oscillations

\section{Introduction}

The non-thermal broadening of coronal spectral lines was first observed by a rocket-borne instrument (Hassler et al. 1990) and more recently by the CDS and SUMER instruments on the SOHO spacecraft (Banerjee et al. 1998; Doyle et al. 1998, 1999; Harrison et al. 2002; O'Shea et al. 2003, 2005). It was found that the Doppler width generally increases with height and this was interpreted as a signature of outwardly propagating undamped Alfvén waves. However, some observations (Harrison et al. 2002; O'Shea et al. 2003, 2005) show a sudden decrease of the line width at an approximate height of $0.1-0.2 R_{\odot}$ above the solar surface. These observations have been performed in coronal structures with different properties (e.g. on the poles and the equator).

It is very important to understand the mechanism of line width decrease as it may trigger the acceleration of plasma particles in these regions. In polar coronal holes, where the magnetic field is open and predominantly vertical, Alfvén waves mainly contribute to the off-limb line broadening due to their transverse velocity polarisation. Acoustic waves propagating along the magnetic field are unlikely to contribute to the line broadening because their velocity polarisation is predominantly perpendicular to the line of sight. Then the decrease of the line width in polar coronal holes can be explained either by the Alfvén wave damping or due to the conversion into acoustic waves. It was first suggested by Hollweg (1971) that the ponderomotive force of linearly or elliptically polarised Alfvén waves may lead to the resonant generation of acoustic waves when the Alfvén and sound speeds become similar (or $\beta=8 \pi p / B^{2} \sim 1$, where $p$ is the plasma pressure and $B$ is the magnetic field). Recently, Zaqarashvili \& Roberts (2006) presented a detailed scenario of wave coupling for $\beta \sim 1$ and showed that Alfvén and sound waves alternately exchange energy when they propagate along a uniform magnetic field with the same phase speed. This means that the Alfvén wave resonantly transfers energy into acoustic waves in a $\beta \sim 1$ plasma and vice versa.

However, the value of the plasma $\beta$ varies with height in the solar atmosphere: it is greater than unity at the photospheric level, but it quickly decreases upwards and becomes smaller in the corona. Nevertheless, it increases again with height and in the solar wind generally $\beta \geq 1$. Therefore, there must be a region in the corona where $\beta$ approaches unity. Recent modelling of the plasma $\beta$ above an active region (Gary 2001) shows that this parameter approaches unity at relatively low coronal heights, of the order of $0.2 R_{\odot}$ (see Fig. 3 in Gary's paper), which surprisingly coincides with the height at which the line width decrease is observed.

Thus, Alfvén waves propagating upwards from the coronal base and entering the region $\beta \sim 1$ can resonantly transfer energy into sound waves, which consequently leads to the observed decrease of the spectral line width near the same height.

In this letter, we present a qualitative analysis of the wave conversion process and compare to the observed decrease of the line width.

\section{Energy conversion from Alfvén to acoustic waves}

We study this process in an open magnetic field configuration using the non-linear, ideal magnetohydrodynamic (MHD) equations written in terms of the fluid velocity, $\boldsymbol{u}$, the magnetic field, $\boldsymbol{B}$, the pressure, $p$, and the density, $\rho$. Gravity is neglected in the equations, as it does not significantly affect the wave interaction process in the corona.

We use a Cartesian coordinate system with the $z$-axis directed radially outward from the Sun. The unperturbed magnetic 
field is directed along the $z$-axis, i.e. $\boldsymbol{B}_{0}=\left(0,0, B_{0}\right)$, and the unperturbed pressure and density, $p_{0}$ and $\rho_{0}$, are allowed to vary in the $z$-direction.

Let us consider wave propagation strictly along the magnetic field, i.e. along the $z$-axis. Then the parallel and perpendicular components of the MHD equations take the form

$\frac{\partial \rho}{\partial t}+\rho \frac{\partial u_{\|}}{\partial z}+u_{\|} \frac{\partial \rho}{\partial z}=0$

$\rho \frac{\partial u_{\|}}{\partial t}+\rho u_{\|} \frac{\partial u_{\|}}{\partial z}=-\frac{\partial p}{\partial z}-\frac{\partial}{\partial z} \frac{b_{\perp}^{2}}{8 \pi}$,

$\rho \frac{\partial u_{\perp}}{\partial t}+\rho u_{\|} \frac{\partial u_{\perp}}{\partial z}=\frac{B_{0}}{4 \pi} \frac{\partial b_{\perp}}{\partial z}$,

$\frac{\partial b_{\perp}}{\partial t}=B_{0} \frac{\partial u_{\perp}}{\partial z}-u_{\|} \frac{\partial b_{\perp}}{\partial z}-b_{\perp} \frac{\partial u_{\|}}{\partial z}$,

$\frac{\partial p}{\partial t}+\gamma p \frac{\partial u_{\|}}{\partial z}+u_{\|} \frac{\partial p}{\partial z}=0$,

where $p$ and $\rho$ denote the total pressure and density, $u_{\|}$and $u_{\perp}$ are the parallel and perpendicular components of the velocity, while $b_{\perp}$ is the magnetic field perturbation. These equations describe the fully non-linear behavior of adiabatic sound and Alfvén waves propagating along an applied magnetic field. The total plasma density and pressure are the sums of the unperturbed and perturbed parts, $\rho_{0}+\rho_{1}$ and $p_{0}+p_{1}$, respectively. Then $u_{\|}$, $\rho_{1}$ and $p_{1}$ are the sound wave velocity, density, and pressure perturbations, whereas $u_{\perp}$ and $b_{\perp}$ are the Alfvén wave velocity and perturbed magnetic field component. We next express perturbations as

$$
\begin{aligned}
& \rho_{1}(z, t)=\tilde{\rho}_{1}(z) \mathrm{e}^{\mathrm{i} \omega_{\mathrm{s}} t}+\tilde{\rho}_{1}^{*}(z) \mathrm{e}^{-\mathrm{i} \omega_{\mathrm{s}} t}, \\
& u_{\|}(z, t)=\tilde{u}_{\|}(z) \mathrm{e}^{\mathrm{i} \omega_{\mathrm{s}} t}+\tilde{u}_{\|}^{*}(z) \mathrm{e}^{-\mathrm{i} \omega_{\mathrm{s}} t}, \\
& p_{1}(z, t)=\tilde{p}_{1}(z) \mathrm{e}^{\mathrm{i} \omega_{\mathrm{s}} t}+\tilde{p}_{1}^{*}(z) \mathrm{e}^{-\mathrm{i} \omega_{\mathrm{s}} t}, \\
& b_{\perp}(z, t)=\tilde{b}_{\perp}(z) \mathrm{e}^{\mathrm{i} \omega_{\mathrm{A}} t}+\tilde{b}_{\perp}^{*}(z) \mathrm{e}^{-\mathrm{i} \omega_{\mathrm{A}} t}, \\
& u_{\perp}(z, t)=\tilde{u}_{\perp}(z) \mathrm{e}^{\mathrm{i} \omega_{\mathrm{A}} t}+\tilde{u}_{\perp}^{*}(z) \mathrm{e}^{-\mathrm{i} \omega_{\mathrm{A}} t},
\end{aligned}
$$

where $\omega_{\mathrm{s}}$ and $\omega_{\mathrm{A}}$ are the sound and Alfvén wave frequencies, correspondingly, and the symbol $*$ denotes the complex conjugate.

Substitution of expressions (6)-(10) into Eqs. (1)-(5) and subsequent time averaging over a time interval much larger than the sound and Alfvén wave periods leads to the cancellation of second order terms, so that only the linear and third order advective terms remain. This means that Alfvén and sound waves are decoupled and that the energy exchange process does not occur. Nevertheless, if the wave frequencies satisfy the condition $\omega_{\mathrm{s}}=2 \omega_{\mathrm{A}}$, then the second order terms also remain and we obtain a set of non-linear differential equations with $z$-derivatives describing the non-linear energy exchange between sound and Alfvén waves. Let us consider the weakly non-linear process in which perturbations are much smaller than the unperturbed values. Then, using the adiabatic relation $p_{1}=c_{\mathrm{s}}^{2} \rho_{1}$ and neglecting third and higher order terms leads to the equations

$$
\begin{aligned}
& \frac{\partial \tilde{\rho}_{1}}{\partial z}=-\frac{2 \mathrm{i} \omega_{\mathrm{A}} \rho_{0}}{c_{\mathrm{s}}^{2}} \tilde{u}_{\|}-\frac{2 c_{\mathrm{s}}^{\prime}}{c_{\mathrm{s}}} \tilde{\rho}_{1}-\frac{\mathrm{i} \omega_{\mathrm{A}} \rho_{0}}{B_{0} c_{\mathrm{s}}^{2}} \tilde{b}_{\perp} \tilde{u}_{\perp}, \\
& \frac{\partial \tilde{u}_{\|}}{\partial z}=-\frac{2 \mathrm{i} \omega_{\mathrm{A}}}{\rho_{0}} \tilde{\rho}_{1}-\frac{\rho_{0}^{\prime}}{\rho_{0}} \tilde{u}_{\|}, \\
& \frac{\partial \tilde{u}_{\perp}}{\partial z}=\frac{\mathrm{i} \omega_{\mathrm{A}}}{B_{0}} \tilde{b}_{\perp}-\frac{2 \mathrm{i} \omega_{\mathrm{A}}}{\rho_{0} B_{0}} \tilde{\rho}_{1} \tilde{b}_{\perp}^{*}-\frac{\mathrm{i} \omega_{\mathrm{A}}}{v_{\mathrm{A}}^{2}} \tilde{u}_{\|} \tilde{u}_{\perp}^{*}-\frac{\rho_{0}^{\prime}}{\rho_{0} B_{0}} \tilde{u}_{\|} \tilde{b}_{\perp}^{*}, \\
& \frac{\partial \tilde{b}_{\perp}}{\partial z}=\frac{\mathrm{i} \omega_{\mathrm{A}} B_{0}}{v_{\mathrm{A}}^{2}} \tilde{u}_{\perp}-\frac{\mathrm{i} \omega_{\mathrm{A}} B_{0}}{\rho_{0} v_{\mathrm{A}}^{2}} \tilde{\rho}_{1} \tilde{u}_{\perp}^{*}-\frac{\mathrm{i} \omega_{\mathrm{A}}}{v_{\mathrm{A}}^{2}} \tilde{u}_{\|} \tilde{b}_{\perp}^{*},
\end{aligned}
$$
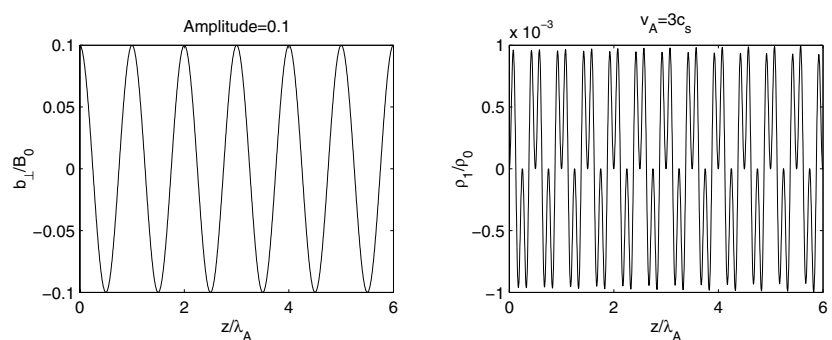

Fig. 1. Spatial dependence of the magnetic field and density perturbations for $v_{\mathrm{A}}=3 c_{\mathrm{s}}$. At $z=0$ there are only Alfvén waves with an amplitude $\tilde{b}_{\perp} / B_{0}=0.1$. The amplitude of Alfvén waves remains unchanged with height: there is no energy transfer into acoustic waves.

where $c_{\mathrm{s}}$ and $v_{\mathrm{A}}$ are the sound and Alfvén speeds and primes denote the derivative with respect to $z$. Equations (11)-(14) describe the time-averaged, weakly non-linear spatial behavior of Alfvén and sound waves propagating along an applied magnetic field.

Let us, for simplicity, first consider an isothermal, homogeneous medium with uniform $\rho_{0}$ and $p_{0}$. If the backreaction of sound waves is neglected (which means that the non-linear terms in Eqs. (13), (14) are neglected), then we get the equation for linear Alfvén waves, which leads to the solutions $\tilde{u}_{\perp}, \tilde{b}_{\perp} \sim \mathrm{e}^{i k_{\mathrm{A}} z}$ and the dispersion relation $\omega_{\mathrm{A}}^{2}=v_{\mathrm{A}}^{2} k_{\mathrm{A}}^{2}$, where $k_{\mathrm{A}}$ is the wave number of Alfvén waves. Thus, in this case the amplitude of Alfvén waves remains constant over the whole spatial domain (this is because we neglected the backreaction of sound waves). Then, the sound wave Eqs. (11) and (12) give

$\frac{\partial^{2} \tilde{u}_{\|}}{\partial z^{2}}+\frac{4 \omega_{\mathrm{A}}^{2}}{c_{\mathrm{S}}^{2}} \tilde{u}_{\|}=-\frac{2 \omega_{\mathrm{A}}^{3}}{B_{0}^{2} k_{\mathrm{A}} c_{\mathrm{s}}^{2}} \tilde{b}_{\perp}^{2}$

This equation is typical of oscillations under the action of an external force and is characterised by having a resonant solution when the wavelength of the external oscillations coincides with the natural oscillatory wavelength of the system. Here the ponderomotive force of Alfvén waves acts as an external periodic driver. Therefore, the sound wave resonant harmonics possess a wave number $2 k_{\mathrm{A}}$ (because of the square of $b_{\perp}$ in Eq. (15)) when $v_{\mathrm{A}} \approx c_{\mathrm{S}}$ is satisfied. Therefore, the ponderomotive force of Alfvén waves may resonantly drive sound waves in the vicinity of the $\beta \sim 1$ region, an effect which to our knowledge was first pointed out by Hollweg (1971).

However, for a comparison with observations, the backreaction of sound waves must be taken into account in order to see the decrease of Alfvén wave amplitude due to the energy conversion. We therefore solve numerically the complete set of Eqs. (11)-(14) with boundary conditions representing the upward propagation of Alfvén waves from the coronal base. We thus impose $\tilde{b}_{\perp} / B_{0}=-\tilde{u}_{\perp} / v_{\mathrm{A}} \neq 0, \tilde{u}_{\|}=\tilde{\rho}_{1}=0$ at $z=0$.

The spatial dependence of the magnetic field and density perturbations for $v_{\mathrm{A}}=3 c_{\mathrm{s}}$ is shown in Fig. 1 . At $z=0$ there are only Alfvén waves with a relative amplitude $\tilde{b}_{\perp} / B_{0}=0.1$ and a wavelength $\lambda_{\mathrm{A}}$, while all perturbations related to acoustic waves are zero. For $z>0$ the ponderomotive force excites density fluctuations with a wavelength smaller than $\lambda_{\mathrm{A}}$, but the amplitude of Alfvén waves is unchanged. In addition, density fluctuations remain small and display no spatial growth, while the modulation with half the wavelength of Alfvén waves can be traced. Thus, there is no resonant energy transfer from Alfvén into acoustic waves. This is a typical behavior of a forced system when the external frequency of oscillation does not coincide with the 

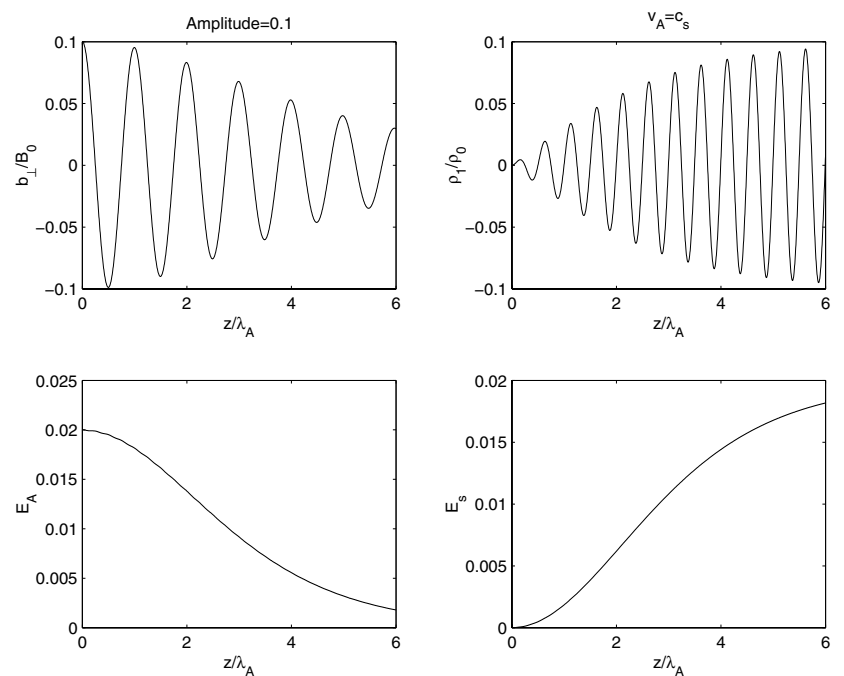

Fig. 2. Spatial dependence of the magnetic field and density perturbations and energy of Alfvén and acoustic waves under resonant conditions, i.e. for $v_{\mathrm{A}}=c_{\mathrm{s}}$. At $z=0$ there are only Alfvén waves with an amplitude $\tilde{b}_{\perp} / B_{0}=0.1$. Because of the resonant process, the energy of Alfvén waves decays with height and density perturbations with half the wavelength of the Alfvén wave are exponentially amplified.

system's natural frequency of oscillation (in the present context the word "frequency" should be substituted by "wavelength").

Next let us consider the case $v_{\mathrm{A}}=c_{\mathrm{s}}$. The spatial dependence of the magnetic field and density perturbations and the energies of Alfvén and acoustic waves are plotted in Fig. 2. At low heights (i.e. for small $z$ ) there are only Alfvén waves with an amplitude $\tilde{b}_{\perp} / B_{0}=0.1$ and acoustic wave perturbations are negligible. At larger heights the energy of Alfvén waves decreases and density perturbations with half the wavelength of Alfvén waves are exponentially amplified. It is clearly seen that the energy of Alfvén waves is effectively transformed into sound wave energy. Moreover, the efficiency of the energy conversion depends on the wave amplitude and the stronger the amplitude of Alfvén waves at $z=0$, the faster the energy conversion process. To show this effect, in Fig. 3 we plot the results for $v_{\mathrm{A}}=c_{\mathrm{s}}$, but now with a larger Alfvén wave amplitude at $z=0$, namely $\tilde{b}_{\perp} / B_{0}=0.3$. The faster decay of the Alfvén wave amplitude is evident: the amplitude of Alfvén waves decreases by almost a factor three in a distance of two wavelengths.

In the case of a spatially inhomogeneous plasma $\beta$ it can be shown that Alfvén waves propagating with almost constant amplitude in the $\beta \neq 1$ region undergo a rapid decay (transferring their energy into density perturbations) when they enter the region $\beta \sim 1$. In this case the height variation of unperturbed parameters (density, temperature, magnetic field) relevant to real conditions in the corona must be given. However this is not the scope of this letter and is thus left for a future study.

Following Gary (2001), the plasma $\beta$ above an active region can approach unity at relatively low coronal heights $(z \sim$ $0.2 R_{\odot}$ ). Outside active regions $\beta$ can approach unity even at lower heights. Hence, Alfvén waves propagating outward from the solar coronal base can efficiently decay and their energy transformed into sound waves in or near the regions where the plasma $\beta$ is close to unity. In polar coronal holes, where a sudden decrease of the Doppler width has been observed (O'Shea et al. 2003, 2005), the open magnetic field is almost vertical. Therefore only the velocity component transverse to the field lines may significantly contribute to the off-limb line
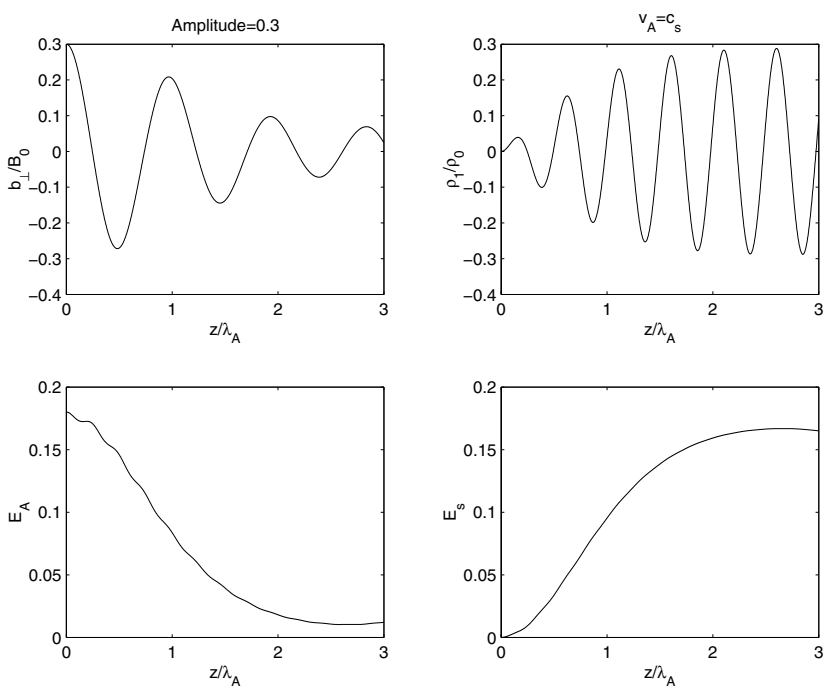

Fig. 3. Same as in Fig. 2, but for the Alfvén wave amplitude $\tilde{b}_{\perp} / B_{0}=0.3$ at $z=0$. The rapid decay of the Alfvén wave in 2-3 wavelengths is clearly seen.

broadening. On the contrary, the velocity component along the field lines will cause a negligible contribution because it is almost perpendicular to the line of sight. Hence the conversion of Alfvén into acoustic waves can be the reason for the observed decrease of the line width in coronal holes. This suggestion is further supported by the agreement between the heights where the spectral line width decrease is observed and where $\beta$ approaches unity. In the ideal case, the energy transferred to acoustic waves will be returned back to Alfvén waves after some distance, although this may not be the case in a more realistic situation since the generated sound waves can be quickly attenuated by Landau damping (or other damping mechanism) leading to the acceleration of plasma particles.

However if the magnetic field is inclined or has a complex structure (for example, above active regions) then the excited acoustic waves may have a velocity component parallel to the line of sight. Then they also will contribute to the line broadening and therefore the line width will not be reduced. This is further supported by the fact that some observations do not show the line width reduction (Wilhelm et al. 2004). So, possibly, the observation of line width reduction depends on the magnetic field orientation in the observed region.

\subsection{Comparison with observations}

Now let us make a comparative analysis between the theoretically obtained decay due to energy conversion and the observed decrease of the line width. Observations (Harrison et al. 2002; O'Shea et al. 2003, 2005) show a similar decay rate of the line width: this quantity decreases by $\sim 10 \%$ over a distance of $\sim 70-100 \mathrm{Mm}$. This means that the amplitude of Alfvén waves is also reduced by $\sim 10 \%$ over the same distance. In order to estimate the decay rate of Alfvén waves, their wavelength must be known. Unfortunately, observations do not reveal neither the wavelength nor the period of propagating Alfvén waves, although some estimations can be done. Let us suppose that the Alfvén wave period is $\sim 5 \mathrm{~min}$. In the region where $\beta \sim 1$, the Alfvén and sound speeds have similar values, i.e. $\sim 200 \mathrm{~km} \mathrm{~s}^{-1}$ (thus, in this region $v_{\mathrm{A}}$ is smaller than its value in the lower corona, $\sim 1000 \mathrm{~km} \mathrm{~s}^{-1}$ ). Then, from $v_{\mathrm{A}} \sim 200 \mathrm{~km} \mathrm{~s}^{-1}$ and assuming a wave period $\sim 5 \mathrm{~min}$, the wavelength is $\lambda_{\mathrm{A}} \sim 60 \mathrm{Mm}$. So 
if the line broadening is caused by the damping of Alfvén waves with a period of $5 \mathrm{~min}$, then their amplitude is reduced by $\sim 10 \%$ over a distance of $1-2 \lambda_{\mathrm{A}} \sim 60-120 \mathrm{Mm}$, in good agreement with observations. The observed spectral line width implies Alfvén wave velocity perturbations of the order of $20-40 \mathrm{~km} \mathrm{~s}^{-1}$ (Harrison et al. 2002). Thus, the relative amplitude (wave velocity divided by phase speed) of Alfvén waves can be estimated as $0.1-0.2$. Hence, for a comparison let us take the theoretical plot for Alfvén waves with relative amplitude of 0.1, i.e. Fig. 2. This figure shows that the Alfvén wave amplitude is reduced by $10 \%$ in $1-1.5$ wavelengths, which is in perfect agreement with observations.

\section{Conclusions}

We show that the resonant energy conversion from Alfvén to sound waves near the region where the plasma $\beta$ approaches unity (or more precisely, where the ratio of sound to Alfvén speeds approaches unity) may explain the observed sudden decrease of the spectral line width in the solar corona. The estimated decay rate of Alfvén waves with period $\sim 5 \mathrm{~min}$ and amplitude 0.1 perfectly fits the observations. However, Alfvén waves with shorter period and smaller amplitude (or longer period and larger amplitude, see Fig. 3) can also explain the observed decay with the same success. Hence, the determination of the period or wavelength of propagating Alfvén waves responsible for the reported line broadening will be a good test for this theory.
Sound waves (or more properly, ion-acoustic waves) may accelerate plasma particles due to Landau damping, which is very efficient when the electron temperature approaches the ion temperature, because the wave phase speed now resides well inside the Maxwellian distribution of particle velocities (Chen 1984), therefore the generated ion-acoustic waves will be quickly damped, transforming their energy into kinetic energy of plasma particles. As a result, the solar wind acceleration may begin near the region of the solar corona where the hydrodynamic and magnetic energy densities approach each other. This requires detailed observational and theoretical study.

Acknowledgements. The work was supported by MCyT grant AYA2003-00123.

\section{References}

Banerjee, D., Teriaca, L., Doyle, J. G., \& Wilhelm, K. 1998, A\&A, 339, 208

Chen, F. F. 1984, Introduction to plasma physics and controlled fusion (London: Plenum)

Doyle, J. G., Banerjee, D., \& Perez, M. 1998, Sol. Phys., 181, 91

Doyle, J. G., Teriaca, L., \& Banerjee, D. 1999, A\&A, 349, 956

Harrison, R. A., Hood, A. W., \& Pike, C. D. 2002, A\&A, 392, 319

Hassler, D. M., Rottman, G. J., Shoub, E. C., \& Holzer, T. E. 1990, ApJ, 348, L77

Hollweg, J. V. 1971, J. Geophys. Res., 76, 5515

Gary, G. A. 2001, Sol. Phys., 203, 71

O'Shea, E., Banerjee, D., \& Poedts, S. 2003, A\&A, 400, 1065

O'Shea, E., Banerjee, D., \& Doyle, J. G. 2005, A\&A, 436, L35

Wilhelm, K., Dwivedi, B. N., \& Teriaca, L. 2004, A\&A, 415, 1133

Zaqarashvili, T. V., \& Roberts, B. 2006, A\&A, 452, 1053 